Results: A total of 49 patients were enrolled and 48 patients completed the study. The mean injection site pain score was 2.3 in PFS vs. 2.0 in PFP immediately post-injection and 0.8 in PFS vs. 0.7 in PFP at $15-30$ minutes post-injection. At both time points the score was equivalent between PFS and PFP: the $97.5 \% \mathrm{Cl}$ was $(-0.99,0.30)$ and $(-0.47,0.25)$ immediately and $15-30$ minutes post-injection, respectively.

The overall impression was also comparable between PFS and PFP. There were no patients who had an overall impression of extremely unfavorable and the proportion of patients who had a favorable impression was higher than that of unfavorable impression in both PFS and PFP. The overall preference for PFP $(56.5 \%)$ was higher than PFS (30.4\%) as presented in the Table.

Both PFS and PFP were well tolerated and there were no serious treatmentemergent adverse events. Only one patient after administration of PFS experienced injection site reaction.

Table. Preference between Pre-filled Syringe and Pre-filled Pen

\begin{tabular}{|l|c|c|c|}
\hline \multicolumn{1}{|c|}{ Preference } & $\begin{array}{c}\text { Pre-filled Syringe } \\
\mathbf{n}^{\prime} \mathbf{n}^{\prime}(\%)\end{array}$ & $\begin{array}{c}\text { Pre-filled Pen } \\
\mathbf{n} / \mathbf{n}^{\prime}(\%)\end{array}$ & $\begin{array}{c}\text { No Preference } \\
\mathbf{n} / \mathbf{n}^{\prime}(\%)\end{array}$ \\
\hline Overall preference & $14 / 46(30.4)$ & $26 / 46(56.5)$ & $6 / 46(13.0)$ \\
\hline Preference categories & & & \\
\hline Ease to use & $10 / 46(21.7)$ & $33 / 46(71.7)$ & $3 / 46(6.5)$ \\
\hline Convenience & $6 / 46(13.0)$ & $35 / 46(76.1)$ & $5 / 46(10.9)$ \\
\hline Time to administration injection & $9 / 46(19.6)$ & $30 / 46(65.2)$ & $7 / 46(15.2)$ \\
\hline Safety & $6 / 46(13.0)$ & $31 / 46(67.4)$ & $9 / 46(19.6)$ \\
\hline Less pain & $17 / 46(37.0)$ & $21 / 46(45.7)$ & $8 / 46(17.4)$ \\
\hline
\end{tabular}

Conclusions: The injection site pain score of PFS and PFP was comparable with overall preference rate higher for PFP. Both PFS and PFP were well tolerated with similar safety profiles.

References:

[1] Shin D et al. Ann Rheum Dis. 2015; 74 (Suppl2: 459-460), FRI0110.

[2] Weinblatt ME et al. Arthritis Rheumatol. 2015; 67 (suppl 10).

[3] Shin D et al. Ann Rheum Dis 2016;75(Suppl2): 1005.

Disclosure of Interest: J. Ghil Employee of: Samsung Bioepis Co., Ltd., J. Niebrzydowski Grant/research support from: Samsung Bioepis Co., Ltd., A. Zielińska Grant/research support from: Samsung Bioepis Co., Ltd., Y. Lee Employee of: Samsung Bioepis Co., Ltd.

DOI: 10.1136/annrheumdis-2017-eular.3350

\section{FRI0199 SYSTEMATIC REVIEW AND META-ANALYSIS ON CERTOLIZUMAB PEGOL FOR RHEUMATOID ARTHRITIS IN ADULTS}

J.A. Bernal ${ }^{1}$, P. Vela ${ }^{1}$, V. Ruiz García ${ }^{2}$, A. Burls ${ }^{3}$, J.B. Cabello ${ }^{4}$, S. Bort-Martí ${ }^{5}$ ${ }^{1}$ Rheumatology, Hospital General Universitario de Alicante, Alicante; ${ }^{2}$ Hospital at Home Unit, la Fe University Hospital, Valencia, Spain; ${ }^{3}$ School of Health Sciences, University of London, London, United Kingdom; ${ }^{4}$ Cardiology \& CASP Spain, Hospital General Universitario de Alicante, Alicante, ${ }^{5}$ Acella Incubator, Paterna, Valencia, Spain

Background: The appearance of tumor necrosis factor-alpha (TNFalpha) inhibitors dramatically changed the prognosis of rheumatoid arthritis. Certolizumab pegol (CZP) is a human Fab fragment of anti-TNFalpha monoclonal antibody which is approved for the treatment of rheumatoid arthritis. We performed a systematic review and meta-analysis, with Cochrane methodology, of the effects of CZP in rheumatoid arthritis.

Objectives: To assess the clinical benefits and harms of CZP in patients with rheumatoid arthritis.

Methods: We performed a search of electronic database (Cochrane Database, MEDLINE, EMBASE, Web of Knowledge and clinicaltrials.gov) until 26th September 2016. We searched for randomized controlled trials of CZP in rheumatoid arthritis compared to any other agent including placebo.

Results: 14 trials were included for the meta-analysis, 12 (5422 patients) in the pooled analysis for benefits and 14 (5499 patients) in the pooled analysis for safety. The overall possibility of bias seemed to be low but the quality of the evidence was low due to the risk of attrition bias.

With the approved dose - CZP $200 \mathrm{mg}$ subcutaneous every other week with the first three doses of $400 \mathrm{mg}$ - CZP showed statistically significant improvements at 24 weeks compared to placebo in: ACR50 absolute improvement $27 \%(95 \% \mathrm{Cl}$ $20 \%$ to $33 \%$ ), RR $3.8(95 \% \mathrm{Cl} 2.42$ to 5.95$)$ and NNT=4 (95\% Cl 3 to 8$)$; DAS28 $<2.6$ - original definition of remission - with RR $3.79(95 \% \mathrm{Cl} 1.90$ to 7.56$)$; $\mathrm{HAQ}$ with $-12 \%$ absolute improvement ( $95 \% \mathrm{Cl}-9 \%$ to $-14 \%$ ); and erosion score with $-0.29 \%(95 \% \mathrm{Cl}-0.42 \%$ to $-0.17 \%)$. There are also data available at 12 weeks with RR of $1.99(95 \% \mathrm{Cl} 1.44$ to 2.76$)$ of achieving DAS28<2.6 with CZP $200 \mathrm{mg}$ dose. The proportion of patients achieving DAS28 $<2.6$ was still higher with CZP at 52 weeks with RR of $1.83(95 \% \mathrm{Cl} 1.53$ to 2.18$)$.

Serious adverse events were more frequent for CZP $200 \mathrm{mg}$ dose with a RR of $1.47(95 \% \mathrm{Cl} 1.13$ to 1.91$)$ and $\mathrm{NNH}$ of 32 . There have been eight adverse events leading to death in CZP $200 \mathrm{mg}$ group versus two in the control group (not statistically significant) and 10 patients developing tuberculosis versus two in the control group (not statistically significant).
Conclusions: There is low level evidence from randomized controlled trials that CZP as monotherapy or combined with methotrexate improved ACR50, DAS28, $\mathrm{HAQ}$ and joint damaged on $\mathrm{x}$-ray. Adverse events were more frequent with active treatment.

Disclosure of Interest: None declared

DOI: 10.1136/annrheumdis-2017-eular.6433

\section{FRI0200 HIGHER ACCEPTANCE AND PERSISTENCE RATES AFTER BIOSIMILAR TRANSITIONING IN PATIENTS WITH A RHEUMATIC DISEASE AFTER EMPLOYING AN ENHANCED COMMUNICATION STRATEGY}

L. Tweehuysen ${ }^{1}$, V.J.B. Huiskes ${ }^{2}$, B.J.F. van den Bemt ${ }^{2,3}$, F.H.J. van den Hoogen ${ }^{1,4}$, A.A. den Broeder ${ }^{1,4}$. ${ }^{1}$ Rheumatology; ${ }^{2}$ Pharmacy, Sint Maartenskliniek; ${ }^{3}$ Pharmacy; ${ }^{4}$ Rheumatology, Radboudumc, Nijmegen, Netherlands

Background: In blinded trials, transitioning from an innovator to a biosimilar has shown to be equivalent to maintenance on innovator biologic treatment in rheumatic diseases. However, data on open label transitioning to a biosimilar are scarce. Recently, we sequentially implemented two biosimilar transition projects (from innovator infliximab (REM) to biosimilar infliximab (CT-P13) and from innovator etanercept (ENB) to biosimilar etanercept (SB4)) in patients with a rheumatic disease using different communication strategies.

Objectives: To investigate the impact of different communication strategies on the acceptance and persistence rates after transitioning from ENB to SB4 in 2016 and transitioning from REM to CT-P13 in 2015.

Methods: Adult patients treated with REM or ENB were informed by letter about the request to transition to a biosimilar. Subsequently, patients were approached by telephone to ask whether they agreed. After the transition of REM to CT-P13 had finished, communication was enhanced for the transition of ENB to SB4 by 1) informing all patients at the same time directly followed by a national media item, 2) reporting that lower costs and fewer injection site reactions (demonstrated in a previous trial) were the reason for transitioning, 3) providing a "soft skills" training for rheumatology and pharmacy staff about how to assuage patient concerns regarding a biosimilar and how to act if a patient has objective or subjective health complaints (discuss possible nocebo response and incorrect causal attribution). Also, group think effects did not play a role during SB4 treatment (individual subcutaneous versus group intravenous administration). Transitioning patients were eligible for inclusion in the BIO-SWITCH study (switch REM to CT-P13) and BIO-SPAN study (switch ENB to SB4) ${ }^{1}$. Demographic, disease and treatment specific characteristics at baseline and disease activity and adverse events (AEs) during 6 months follow-up were collected.

Results: 196 of $222(88 \%)$ REM-treated patients and 636 of $643(99 \%)$ ENBtreated patients transitioned to respectively CT-P13 and SB4 (delta $11 \%, 95 \% \mathrm{Cl}$ $7 \%$ to $16 \%$ ). Until January 1 st 2017,479 of $636(75 \%)$ patients gave informed consent for the BIO-SPAN study. Baseline characteristics were similar, except for disease duration and innovator treatment duration (both shorter in the BIO-SPAN cohort; $p<0.01$ ). Drug survival of patients on SB4 was adjusted for confounders greater than patients on CT-P13 (Figure 1; adjusted Hazard Rate 0.21, 95\% $\mathrm{Cl} 0.13$ to 0.34 ). During 84 person-years of follow-up 47 patients discontinued CT-P13 (56/100 person-years; $26 \%$ due to inefficacy, $74 \%$ due to AEs). In contrast, 36 patients discontinued SB4 during 230 person-years of follow-up (16/100 person-years; $53 \%$ due to inefficacy, $42 \%$ due to AEs and $5 \%$ due to remission).

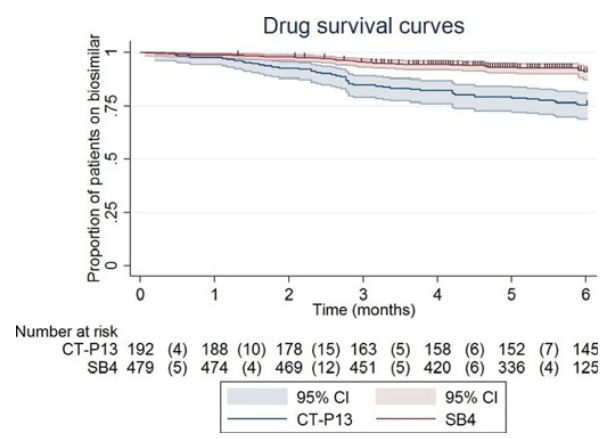

Conclusions: Use of an enhanced communication strategy, together with more experience and absence of group think effects, resulted in much higher acceptance and persistence rates after open label shared decision making biosimilar transitioning in patients with a rheumatic disease.

Acknowledgements: This study was funded by Biogen

Disclosure of Interest: L. Tweehuysen: None declared, V. Huiskes: None declared, B. van den Bemt Speakers bureau: Pfizer, AbbVie, Mundipharma, F. van den Hoogen Consultant for: Biogen, Celltrion, Janssen, Mundipharma and Sandoz, A. A. den Broeder Consultant for: AMGEN

DOI: 10.1136/annrheumdis-2017-eular.2889 